\title{
Comprendre et accompagner les élèves en difficulté de lecture au début du secondaire. Echos d'une recherche-action en didactique de la lecture littéraire
}

Qui sont les élèves réputés " faibles » et " mauvais » lecteurs si on les considère dans leur diversité et dans leur singularité ? Comment expliquer que certains adolescents ne comprennent pas ce qu'ils lisent? Plus précisément, que comprennent les élèves en difficulté de lecture quand ils lisent? Quels modes de lecture mettent-ils en œuvre quand ils s'efforcent de comprendre ou d'interpréter des textes littéraires ? Quelles sont les réponses didactiques les plus adaptées aux difficultés des élèves? Telles sont les questions qui constituent tout à la fois le moteur, la trame et le sens de la recherche doctorale dont il est question dans cette contribution.

\section{UNE RECHERCHE-ACTION EN DIDACTIQUE DE LA LECTURE LITTÉRAIRE À DESTINATION DES}

\section{ÉLÈVES EN DIFFICULTÉ AU DÉBUT DU SECONDAIRE}

La recherche cible les élèves présentant un déficit spécifique de la compréhension au début de l'enseignement secondaire. Elle vise une double finalité compréhensive et praxéologique: le projet de recherche est pensé en termes d'apports à l'analyse des difficultés et à l'outillage des pratiques enseignantes. Ces deux objectifs dialectiquement articulés débouchent sur une recherche qualitative, longitudinale et écologique, dont le format consiste en une recherche-action visant à accompagner, sur deux années scolaires, dix élèves identifiés en difficulté de compréhension au début $\mathrm{du} 1^{\mathrm{er}}$ degré différencié ${ }^{1}$ et à observer ces élèves in situ, dans le contexte naturel de la classe, pour les décrire qualitativement. La recherche consiste en outre en une intervention collaborative à dispositif ouvert: un programme d'accompagnement multidimensionnel a été mis au point en collaboration avec les deux enseignantes partenaires et ajusté d'une étape à l'autre en fonction des effets constatés. Le programme d'intervention mis en œuvre repose sur différentes hypothèses ${ }^{2}$ à propos des réponses didactiques qui paraissent les plus appropriées pour aider les lecteurs en difficulté. La lecture littéraire, $1^{\text {er }}$ postulat, peut remobiliser les élèves à l'égard de la lecture. Font aussi partie des postulats de recherche relatifs à l'aide aux lecteurs en difficulté (1) la clarification, pour l'apprenant, de la lecture en tant qu'activité et de ses propres procédures de lecteur, ainsi que (2) le développement de l'estime de soi et du sentiment de sécurité lecturale. Enfin, l'aide aux " mauvais lecteurs" passe par une clarification, pour l'enseignant, des dispositifs de problématisation des textes et d'évaluation de l'activité de compréhension des lecteurs.

Prenant pour appui sur la typologie des profils de mauvais " compreneurs " élaborée par Van grunderbeeck et Payette (2007), l'échantillon de sujets se déploie de la façon suivante.

- Un $1^{\mathrm{er}}$ groupe d'élèves rassemble les élèves trop centrés sur les microprocessus (qui ne sont pas tout à fait automatisés) et ne parviennent pas à dégager l'essentiel du texte.

- D'autres élèves ne saisissent pas les liens sous-jacents : c'est principalement l'inférence qui leur pose problème, en particulier lorsqu'elle se fait complexe et porte sur la globalité du texte.

- Un $3^{\mathrm{e}}$ groupe réunit des lecteurs dont la compréhension est trop globale : ils prélèvent quelques indices épars et s'aident de leurs connaissances pour élaborer un

\footnotetext{
${ }^{1}$ Ce degré accueille les élèves qui sortent du primaire sans avoir obtenu leur Certificat d'Etudes de base.

${ }^{2}$ Ces hypothèses, je les partage avec l'équipe de Catherine Tauveron dont la recherche sur la lecture littéraire comme « voie possible de (ré)conciliation des élèves en difficulté avec la lecture » au sein de l'enseignement fondamental a beaucoup influencé la mienne (Tauveron, 2004).
} 
$1^{\text {er }}$ scénario; mais s'échappent fréquemment dans le hors texte (la lecture peut devenir parfois re-création abusive, au-delà des droits du texte).

- Enfin, des élèves comprennent relativement bien les textes qui leur sont proposés ; ils procèdent aux inférences nécessaires et dégagent aisément l'essentiel d'un texte. C'est du côté des processus métacognitifs que des déficits sont perceptibles. Ces élèves éprouvent des difficultés à verbaliser leurs démarches et à justifier leurs propositions par un retour circonscrit au texte $\left(4^{\mathrm{e}}\right.$ groupe non repris à la typologie des chercheuses québécoises).

\section{EVOCATION DE QUELQUES RÉSULTATS DE LA RECHERCHE}

\subsection{Comprendre les élèves en difficulté de lecture au début du secondaire}

Au terme des deux années d'accompagnement, les élèves du $1^{\text {er }}$ profil connaissent des cheminements variables. Le déficit d'automatisation des microprocessus tend à se maintenir, sans amélioration, chez certains élèves; mais il s'estompe largement chez d'autres. Ce groupe d'élèves est celui qui a le plus maille à partir avec la perception du sens des tâches et des apprentissages en cours : cette difficulté se traduit par un manque de persévérance ou par un " rapport de conformité » aux consignes (Bonnéry, 2007). Les élèves de ce $1^{\text {er }}$ profil rencontrent en conséquence des difficultés à manifester leur compréhension, à fortiori au sein de médiations écrites formalisées. Ce sont eux aussi qui apparaissent les moins "réassurés » dans leur rapport à la lecture.

Les élèves $\mathrm{du} 2^{\mathrm{e}}$ profil sont en évolution à tous les points de vue : non seulement, le déficit dans l'effectuation des liens sous-jacents et dans le traitement des inférences a été comblé, mais on note une familiarité plus grande avec les postures de la lecture littéraire et une plus grande flexibilité dans leur adoption. Les représentations de ces élèves à propos de l'acte de lire se sont sensiblement ajustées et complexifiées.

Les élèves du $3^{\mathrm{e}}$ profil se caractérisent par des trajectoires différentes : le déficit de prise en compte des indices textuels pour corriger les débordements ou les errements d'une compréhension trop globale est comblé chez deux lectrices sur trois, mais il se maintient chez l'une d'elles. L'activité de lecture ne s'est que partiellement clarifiée chez ces élèves.

Enfin, les élèves du $4^{\mathrm{e}}$ profil connaissent une évolution tout à fait significative dans leur capacité à justifier leurs propositions au départ d'indices textuels précisément sélectionnés, mais aussi dans leurs connaissances et leurs représentations de l'activité de lecture. Les avancées sur le plan de la métacognition s'accompagnent d'une évolution de la compréhension et de la capacité à manifester cette compréhension.

En définitive, les trajectoires des élèves sont aussi variables que ne l'étaient les profils de difficulté au départ. Le $1^{\text {er }}$ profil connait la progression la moins marquée. Les élèves les plus en difficulté en début d'intervention semblent progresser moins significativement que les autres. Le déficit de connaissances métacognitives au départ semble ainsi freiner la progression (Rémond, 2001). À l'inverse, les élèves du $4^{\mathrm{e}}$ profil, celui des lecteurs presque confirmés présentant une difficulté à l'égard de la métacognition, connaissent sous l'influence d'un programme d'entrainement centré prioritairement sur ces compétences-là, une forte progression tant dans le domaine de la compréhension que dans celui de la métacognition. Cet entrainement à visée métacognitive profite en outre aux élèves du $2^{\mathrm{e}}$ profil, et, de façon plus variable, aux élèves du $3^{\mathrm{e}}$ profil.

\subsection{Accompagner les élèves en difficulté de lecture au début du secondaire}


- $\quad$ Retour sur un 1er postulat de la recherche : la lecture littéraire peut contribuer à remobiliser les lecteurs en difficulté

La fiction semble confirmée dans sa fonction d'enrôlement des élèves dans la lecture. Certains élèves semblent avoir davantage pris conscience des profits symboliques qu'ils pouvaient tirer de la lecture de la littérature - ceci n'impliquant pas que ces élèves soient devenus des « gros » lecteurs.

Relativement aux différentes " catégories » de textes exploités dans le cadre des dispositifs de la recherche-action, il apparait que la fictionnalisation d'expériences de lecteurs (dans des textes métanarratifs, des scènes et images de la lecture) constitue une alternative didactique intéressante à l'explicitation au départ de l'action propre pour amener les élèves à construire des connaissances sur la lecture et à développer la conscience des stratégies efficaces pour comprendre les textes. Les textes résistants semblent quant à eux avoir favorisé l'adoption d'une double posture de collaboration et de méfiance, d'un " rapport à la fiction » propice à la pratique du jeu de la lecture littéraire. Ils ont contribué au développement de certains processus de lecture (en particulier l'inférence). Enfin, ils ont joué un rôle favorable à l'engagement dans la lecture et dans les échanges.

Ces effets positifs paraissent toutefois conditionnés, notamment par le recours aux interactions tant avec les pairs qu'avec l'enseignant. En effet, la persévérance dans la lecture et le traitement des données inférentielles reposent, outre sur la brièveté des textes qui permet de limiter les problèmes de mémorisation dans un $1^{\text {er }}$ temps, sur les échanges avec les pairs et avec l'enseignant.

- $\quad$ Retour sur un $2^{\mathrm{e}}$ postulat de la recherche : l'aide aux lecteurs en difficulté passe par une clarification, pour l'apprenant, de l'(son) activité de lecture

Les moments de "retour réflexif " sur les activités menées ont eu un effet bénéfique plusieurs élèves manifestent, en discours et en actes, des représentations (en partie) clarifiées en fin d'intervention - mais pas aussi étendu qu'escompté. Ces moments ont contribué à la clarification de la nature de l'activité de lecture dans la mesure où ils ont permis aux élèves d'identifier des difficultés de lecture et, en regard de celles-ci, des procédures qui permettent de les dépasser. Les tâches d'introspection, des écrits réflexifs pour l'essentiel (tels que l'autoportrait ou l'autobiographie de lecteur/lectrice), ont débouché sur des bénéfices plus nettement perceptibles. Ces tâches ont enrôlé l'ensemble des élèves et leur ont visiblement permis d'amorcer la construction d'une image d'eux-mêmes en tant que lecteurs.

L'impact éventuellement positif des interventions à dimension métacognitive semble corrélé à l'alternance entre démarche instrospective individuelle et explicitation collective des observations, ainsi qu'à l'étayage précis de l'enseignant pour rendre perceptibles les apprentissages spécifiques qui se jouent à travers elles.

- Retour sur un $3^{e}$ postulat de la recherche : l'aide aux lecteurs en difficulté passe par une clarification, pour l'enseignant, des dispositifs de problématisation des textes et d'évaluation de la compréhension

\section{Des dispositifs pour problématiser les textes}

Tout d'abord, l'alternance entre la pratique de la lecture comme acte global et le travail, plus local, d'une opération de lecture particulière, isolée pour être amenée à la conscience, 
explicitée et entrainée spécifiquement, semble avoir eu un impact positif sur le plan de la perception de la nature de l'activité de lecture.

Certains des dispositifs mis en œuvre ont paru particulièrement adéquats pour stimuler l'attention des lecteurs aux indices du texte et pour développer la conscience du caractère cyclique de la lecture en tant que processus. C'est souvent la combinaison de deux dispositifs différents, proposés sur un même texte, qui s'est révélée porteuse : il s'est avéré bénéfique d'associer à une démarche de dévoilement progressif d'un récit, une activité de lecture rétroactive (cette dernière permettant de corriger les "sur-interprétations ») ou de prolonger une activité de conception d'un scénario de fin par une tâche de reformulation de l'interprétation finale. Autre tandem qui, dans les dispositifs d'aide aux lecteurs en difficulté, a semblé pertinent: le recours simultané aux échanges de classe et aux écrits de travail individuels. Arrêtons-nous un instant sur ces deux « outils ».

Du côté des interactions, le dialogue des élèves autour du texte a vigoureusement stimulé l'attention des élèves aux indices du texte, mais il a aussi incité ces derniers à opérer les vérifications dont ils ne ressentaient pas le besoin pour eux-mêmes. Ainsi, les échanges de classe ont, plus d'une fois, favorisé le déploiement multiple du dialogue solitaire du lecteur avec le texte. Les élèves se sont révélés davantage capables, progressivement, de partager leurs impressions et leurs hypothèses de lecture, ainsi que de coconstruire et de faire évoluer leurs interprétations. Ces effets bénéfiques des interactions paraissent toutefois conditionnés par plusieurs paramètres : une problématisation individuelle préalable du texte par écrit - les écrits de travail personnels vont nourrir la problématisation collective et aider ensuite à la réappropriation de la parole collective - mais aussi la perception des finalités assignées aux échanges, grâce à un étayage constant de l'enseignant pour centrer l'attention des élèves sur les apprentissages visés par les interactions.

A côté des échanges entre pairs, d'autres types d'interactions se sont avérés bénéfiques au développement de l'engagement, de la clarté cognitive et de la compréhension : ce sont les interactions avec l'adulte Plus précisément, l'étayage de l'adulte, qui, lors d'un entretien de type métacognitif, cherche à comprendre les conduites de lecture d'un élève sur un texte précis, semble favoriser chez le jeune lecteur la conscience des procédures efficaces pour comprendre.

Au final, on pourrait ainsi juger pertinent de développer, chez les élèves en difficulté de compréhension au début du secondaire, trois formes complémentaires de dialogues autour du texte : celui de l'élève seul, celui de collectifs d'élèves, celui de l'élève et de l'enseignant en tête à tête.

Les écrits de travail - divers textes personnels réalisés au cours et au terme de la lecture ont exercé, en interaction avec les échanges, un effet bénéfique sur le développement de la compréhension chez les sujets suivis. Certes, j'ai pu observer chez certains lecteurs une difficulté récurrente dans l'activité de verbalisation écrite de la compréhension. Mais, en dépit de ces obstacles qu'il importe de ne pas méconnaitre, certains écrits d'accompagnement de la lecture ont amené les lecteurs à questionner le texte, mais aussi leur lecture du texte et leur ont ainsi permis de rendre plus intelligible, pour eux-mêmes, leur propre activité de compréhension. En général, en cours de lecture, les écrits de travail gagnent à rester souples, peu contraignants dans leur forme et dans leurs consignes.

Enfin, les dispositifs didactiques mis en œuvre impliquaient un certain nombre de tâches centrées tour à tour sur les attitudes de compréhension (des tâches de reformulation, telle la table des matières commentée...), d'interprétation (des tâches de production d'hypothèses...), d'implication et d'appréciation (telle la tâche de présentation d'un roman pour le catalogue d'une maison d'édition...). Au terme de la lecture, les médiations stimulantes, assimilées à un projet à réaliser, ont favorisé l'engagement des élèves et stimulé leur envie de se parler du texte et d'en parler aux autres. L'inconvénient est que, bien souvent, ces médiations-là impliquent un cadrage plus étroit (en raison du prérequis relatif à la maitrise de normes, notamment génériques). Le cumul d'opérations cognitives de haut 
niveau a empêché certains lecteurs de manifester (toute) leur compréhension. Ainsi, certaines tâches ont pâti d'un manque d'explicitation des visées ou d'un degré de standardisation trop élevé. D'autres, cadrées de façon diffuse, ont posé des problèmes de centration de l'attention en amont, mais ont parfois favorisé l'implication subjective des lecteurs dans les textes. Standardisation et cadrage semblent donc à penser dans des relations d'équilibrage réciproque et dans un souci de progression.

\section{Des dispositifs pour évaluer la compréhension}

Parmi les outils de recueil de données, certaines tâches - l'entretien métacognitif, le journal de lecture, les écrits introspectifs, les verbalisations orales et les diverses traces écrites collectées en cours de lecture... - se sont révélées plus pertinentes, pour recueillir des données sur la compréhension des élèves, que le questionnaire sur les pratiques de lecture, qui souffre trop des biais liés à la désirabilité et préoriente le discours que l'élève tient sur ses propres procédures. Le questionnaire de lecture s'est avéré utile lorsqu'il était complété par des médiations plus " ouvertes » ou plus " globales » de la lecture et lorsqu'il comportait des items qui amenaient le sujet à faire part de son appréciation du texte, à reformuler de façon personnelle le sens global. Les reformulations personnelles finales se sont plus d'une fois avérées nécessaires pour débusquer des problèmes de compréhension qui n'étaient pas apparus ailleurs.

Quant à l'analyse des données recueillies, elle gagne à se construire au départ d'une multiplicité de données, pour un même lecteur, relatives à un même texte (cela permet d'estomper l'effet " tâche " dans la manifestation de la compréhension) et au départ d'une multiplicité de données, pour un même lecteur, relatives à plusieurs textes différents (cela permet d'estomper l'effet " texte " dans l'évaluation des compétences de lecture ou de l'engagement dans la lecture). Elle gagne aussi à mettre en relation les différentes composantes de l'activité du lecteur: la compréhension/interprétation, l'identification des mots, l'engagement, la métacognition et l'appréciation des textes - ceci afin d'éviter d'ériger trop vite une difficulté donnée au rang de "source unique " des faibles performances de lecture d'un élève.

\section{Bibliographie}

BONNERY, St. (2007), Comprendre l'échec scolaire. Elèves en difficultés et dispositifs pédagogiques, Paris, La Dispute.

REMOND, M. (2001), « De l'identification des difficultés de compréhension à l'apprentissage : parcours de recherche au cycle III », in TAUVERON, C. (dir.), Comprendre et interpréter le littéraire à l'école et au-delà, Paris, INRP, 115-123.

TAUVERON, C. (2004), "La lecture littéraire, voie possible de (ré)conciliation des élèves en difficulté avec la lecture ", in TAUVERON, C. (dir.), La lecture et la culture littéraires au cycle des approfondissements. Actes de l'Université d'Automne. Scéren-CRDV de Versailles/Ministère de l'Education nationale, de l'Enseignement Supérieur et de la Recherche, 56-70.

VAN GRunderbeeck, N. et PAyetTe, M. (2007), « Portraits de lecteurs faibles en compréhension au début du secondaire », in Repères, $\mathrm{n}^{\circ} 35,73-92$.

De Croix, S. (2011), «Comprendre et accompagner les élèves en difficulté de lecture au début du secondaire. Echos d'une recherche-action en didactique de la lecture littéraire ", in La Lettre de l'Association internationale pour la Recherche en Didactique du Français, n49, 6-10. 